\section{The impact of urban violence on mental health}

\author{
O impacto da violência \\ urbana na saúde mental
}

Violence is a complex phenomenon involving individuals, interpersonal relationships, communities, and society. Nearly 1.6 million people lost their lives in the year 2000 because of violence, which was the leading cause of death among those aged 15-44 years, having most of the deaths (90\%) occurred in low- and middle-income countries. There are many well known factors which influence worldwide rates of violence: biological, social, cultural, economic, and political. Regarding homicides, 520,000 people were killed in the globe, a rate of 8.8 per 100,000. In Latin America, Colombia and El Salvador present the highest rates of homicides, above 30/100,000 inhabitants, while Brazil, Mexico and Venezuela present rates between 20 and 30/100,000 inhabitants. ${ }^{1}$ Lower levels of homicide rates are seen in Argentina, Chile, Uruguay, and Paraguay.

In Brazil, injury deaths, including traffic accidents and suicides, account for $10 \%$ of the global burden of diseases: homicides alone being responsible for $40 \%$ of the injuries, leading to a $3.0 \%$ yearly loss in the GDP. These all are predictable and preventable deaths. For instance, nearly 50,000 people were murdered in Brazil in the year 2003. Ninety percent of the homicides are perpetrated with firearms in the large urban centers, such as Recife, Rio de Janeiro, Vitória, Cuiabá, and São Paulo. Most of the victims are young, blacks, poorly educated, and raised in deprived areas. Victims and perpetrators share the same tragic destiny: young males are both victims and perpetrators of their acts. Nonetheless, homicide is only a tiny part of the matrix spectrum of violence once many violent events are part of daily life: partner intimate violence, sexual abuse in infancy and adolescence, unwanted pregnancies, forced prostitution, early use of alcohol and drugs, drug trafficking, abuse of the elderly, kidnappings, and blackmail phone calls.

Coexistence in a society embedded in a culture of assaults, aggressions and prejudice, brings a permanent sense of fear and despair, a potent risk factor for mental disorders development either as a trigger or as a developmental component. The impact of the many forms of child and adolescence abuse on mental health is well known. There are strong correlations between early abuse and the occurrence of many psychiatric disorders: major depressive disorder, eating disorders, schizophrenia, bipolar disorder, drug and alcohol dependence, and personality disorders. It is well demonstrated by experimental and clinical studies that adverse events on childhood and adolescence can provoke biological damage in the endocrine, metabolic, immune, and neurophysiology systems.

The extent and nature of the impact of violence on rates of Post-Traumatic Stress Disorder (PTSD) and other common mental disorders, as a consequence of living in the large urban centers on Latin America is unknown, but is expected to be extremely high. It is certain that many cases of PTSD are misdiagnosed and/or missed by our mental health professionals, and remain untreated in the community. These cases are frequently accompanied by depression, panic, substance abuse or psychotic disorders, and mental health professionals are advised to routinely screen for previous trauma 
exposition in their clinical practice. Moreover, from the public health perspective, it is essential to provide services that can minimize sufferance, either at the secondary level, by providing care to victims immediately after the occurrence of the trauma, and at the tertiary level, to reduce disability for those who develop PTSD. Cognitive and exposure therapies are shown to be effective, and SSRIs can be part of an adjunctive treatment. ${ }^{2}$

At the primary prevention level, many challenges lie ahead for future theory and research. One of the most intriguing questions in the traumatology field arises from empirical data showing the existence of important individual differences in how people react to a traumatic event. For instance, although a large number of persons are exposed to violence, not every survivor develops mental disorders. This wellestablished fact has led researchers to try to identify variables that can explain such huge individual differences. This endeavor gave rise to the concept of "resilience", which is an interactive construct that refers to a relative resistance to environmental risk experiences, or the overcoming of adversity. Due to the complexity intrinsic to the resilience field, interdisciplinary research designed within a developmental framework is needed in order to understand the underlying process by which this phenomenon occurs. The recent collaboration between developmental neuroscience and molecular genetics to investigate resilience illustrates the potential for crosscollaboration in this field. ${ }^{3}$

Additionally, future research must continue to investigate the devastating impact of interpersonal violence on children development. Society must be educated about what we know in regard to violence-related neurodevelopmental changes and the profound negative functional consequences of these modifications in the child's brain. ${ }^{4}$ Violence can permanently impair the development of the brain by altering neurogenesis, migration synaptogenesis, and neurochemical differentiation. This fact has implications for clinical assessment, research, intervention, and prevention. The time course of development of the brain is the key to grasp the relationship between exposure to violence in infancy and the emergence of psychopathology. The child's brain is extremely plastic and after puberty a window of opportunity to act decisively to prevent psychopathology may be closed. There is the need for a greater research effort in designing treatment interventions that do not focus only on treating symptoms but also in restoring the child brain's disordered trajectory along a normal course of development. ${ }^{5}$

Jair de Jesus Mari

Department of Psychiatry, Universidade Federal de São Paulo (Unifesp), São Paulo (SP), Brazil Centre for Public Mental Health, Health Services and Population Research Department, Institute of Psychiatry, King's College, University of London, London, UK

Marcelo Feijó de Mello Violence and Posttraumatic Stress Disorder Program, Department of Psychiatry, Universidade Federal de São Paulo (Unifesp), São Paulo (SP), Brazil

Ivan Figueira Institute of Psychiatry, Universidade Federal do Rio de Janeiro (UFRJ), Rio de Janeiro (RJ), Brazil

Disclosures

\begin{tabular}{|c|c|c|c|c|c|c|c|}
\hline $\begin{array}{l}\text { Writting group } \\
\text { member }\end{array}$ & Employment & $\begin{array}{c}\text { Research } \\
\text { grant }^{1}\end{array}$ & $\begin{array}{c}\text { Other research grant or } \\
\text { medical continuous education }\end{array}$ & $\begin{array}{l}\text { Spekear's } \\
\text { honoraria }\end{array}$ & $\begin{array}{l}\text { Ownership } \\
\text { interest }\end{array}$ & $\begin{array}{c}\text { Consultant/ } \\
\text { Advisory board }\end{array}$ & Other ${ }^{3}$ \\
\hline Jair de Jesus Mari & UNIFESP & $\begin{array}{l}\text { FAPESP } \\
\text { CNPq }\end{array}$ & - & - & - & - & - \\
\hline $\begin{array}{l}\text { Marcelo Feijó de } \\
\text { Mello }\end{array}$ & UNIFESP & $\begin{array}{l}\text { FAPESP } \\
\text { CNPq }\end{array}$ & - & - & - & - & - \\
\hline Ivan Figueira & UFRJ & $\begin{array}{l}\text { FAPERJ } \\
\text { CNPq }\end{array}$ & - & - & - & - & - \\
\hline
\end{tabular}

* Modest

** Significant

*** Significant. Amounts given to the author's institution or to a colleague for research in which the author has participation, not directly to the author

Note: UNIFESP = Universidade Federal de São Paulo; UFRJ = Universidade Federal do Rio de Janeiro; CNPq = Conselho Nacional de Desenvolvimento Cientifico e Tecnológico; FAPESP = Fundação de Amparo à Pesquisa do Estado de São Paulo; FAPERJ = Fundação de Amparo à Pesquisa do Estado do Rio de Janeiro.

For more information, see Instructions for authors.

References

1. World Health Organization. World report on violence and health. World Health Organization. Geneva; 2004

2. Mendes DD, Mello MF, Ventura P, Passarela CM, Mari JJ. A systematic review on the effectiveness of cognitive behavioral therapy for posttraumatic stress disorder. Int J Psychiatry Med. 2008; in press.

3. Cicchetti D, Blender JA. A multiple-levels-of-analysis perspective on resilience: implications for the developing brain, neural plasticity, and preventive interventions. Ann N Y Acad Sci. 2006;1094:248-58.

4. Perry BD. The neurodevelopmental impact of violence in childhood. In: Schetky DH, Benedek EP, editors. Textbook of Child and Adolescent Forensic Psychiatry. Washington (DC): American Psychiatric Press; 2001. p. 221-38.

5. Andersen SL. Trajectories of brain development: point of vulnerability or window of opportunity? Neurosci Biobehav Rev. 2003;27(1-2):3-18. 